\title{
The effect of lactobacillus gaseeri THT 031301 supplementation on the body composition and inflammation in adults: pilot study
}

\author{
H. Hamdallah ${ }^{1}$, D. Lucas ${ }^{2}$, B. Kestecher ${ }^{3}$ and N. Abdel Azim ${ }^{1}$ \\ ${ }^{1}$ Chester Medical School, University of Chester, Chester, UK, \\ ${ }^{2}$ Faculty of Health, Liverpool John Moores University, Liverpool, UK and \\ ${ }^{3}$ Faculty of Medicine, Semmelweis University, Budapest, Hungary
}

Human guts are occupied with 100-1000 microbial species which plays a significant role in the host health and disease ${ }^{(1)}$. Lactobacillus species have shown a paradoxical effect in relation to body composition. Clinical trials using lactobacillus gasseri (L. gasseri) have demonstrated an anti-obesity effect, exhibiting significant reductions in visceral and subcutaneous fat, body weight, body mass index (BMI), and waist and hip circumferences ${ }^{(2)}$. Moreover, L. gasseri has contributed to the regulation of abdominal obesity and showed a potential immunomodulatory effect in infants as well as boosting the immune system of healthy adults ${ }^{(3,4)}$. Rodent models have found an association between the presence of L. gasseri and positive outcomes in relation to weight loss, fasting blood glucose $(\mathrm{FBS})^{(5)}$, and inflammation ${ }^{(6)}$. The aim of this study to look at the effect of 4 weeks of supplementation with L. gasseri THT 031301 on obesity, glycaemic, and inflammatory markers.

This is a single centre, double-blind, randomised, placebo-controlled pilot study that recruited fourteen adult subjects with BMI $>25 \mathrm{~kg} / \mathrm{m} 2$, who were randomly assigned to receive either a supplement containing L. gasseri THT $031301(\mathrm{n}=6)$ or a placebo $(n=7)$. Pregnant and lactating women, subjects on anti-diabetic medications, and subjects who have undergone heart surgery have been excluded. Consented subjects were asked to consume 2 capsules per day continuously for 4 weeks. L. gasseri THT 031301 daily dosage was approximately 6x 109cfu. Alterations in obesity markers were assessed using BMI and waist-to-height ratio (WtHR), and inflammatory markers were measured using enzyme-linked immunosorbent assay (ELISA). FBS level, HBA1c, and insulin measured to indicate the glycaemic markers. Each marker measured at baseline and after 4 weeks.

After 4-weeks supplementation, a significant reduction in waist circumference $(\mathrm{WC})(\mathrm{P}=0.022)$ and $(\mathrm{WtHR})(\mathrm{P}=0.035)$ was identified in the THT 031301 compared to the placebo. Within-group comparisons attributed this finding to a significant increase in WC $(93.4 \pm 10.1$ vs $94.6 \pm 9.5, \mathrm{P}=0.040)$ and WtHR $(0.55 \pm 0.04$ vs $0.56 \pm 0.04, \mathrm{P}=0.047)$ in the placebo group. THT 031301 identified a small increase in the anti-inflammatory cytokine IL-10; however, this was not statistically significant. No changes were identified with the glycaemic markers HbA1c, fasting blood glucose, or insulin.

The current pilot study suggests a potential anti abdominal obesity effect of L. gasseri THT 031301 on the glycaemic markers in healthy adults, but this effect needs to be investigated in a large adequately powered RCT. The study did not find a significant effect of L. gasseri THT 031301 on the glycaemic markers. Future studies might recruit obese and overweight subjects or subjects with diabetes to confirm the anti-obesity, anti-diabetic and anti-inflammatory effect of L. gasseri THT 031301.

\section{Acknowledgements}

We would like to thank all the participants for taking part in this trial.

\section{References}

1. Kerry RG, Patra JK, Gouda S, et al. (2018) J Food Drug Anal, 26(3), 927-939.

2. Olivares M, Díaz-Ropero MP, Gómez N et al. (2006) Int Microbiol 9(1), 47-52.

3. Oh NS, Joung JY, Lee JY \& Kim Y. (2018) PloS one 13(2), e0192021.

4. Kadooka, Y., Sato, M., Imaizumi, K et al. (2010) Eur J Clin. Nutr 64(6), 636-643.

5. Million M, Angelakis E, Paul M, et al. (2012) Microb Pathog. 53(2),100-8.

6. Yun SI, Park HO, Kang JH (2009) J Appl Microbiol 107(5), 1681-6. 